\title{
Noninvasive clinical predictors of portal hypertensive gastropathy in patients with liver cirrhosis
}

\author{
Rajesh Mandhwani' ${ }^{1}$, Farina M. Hanif ${ }^{1}$, Muhammad Manzoor Ul Haque', \\ Rajesh Kumar Wadhwa ${ }^{1}$, Nasir Hassan Luck ${ }^{1}$, Muhammad Mubarak ${ }^{2}$ \\ ${ }^{1}$ Departments of Hepatogastroenterology, Sindh Institute of Urology and Transplantation, Karachi, Pakistan; \\ ${ }^{2}$ Departments of Histopathology, Sindh Institute of Urology and Transplantation, Karachi, Pakistan
}

\section{ABSTRACT}

Background and Objectives: Portal hypertensive gastropathy (PHG) is described endoscopically as "mosaic-like appearance" of gastric mucosa with or without the red spots. It can only be diagnosed by upper gastrointestinal $(\mathrm{GI})$ endoscopy. The aim of this study was to determine the diagnostic accuracy of platelet count to spleen diameter ratio (PSR) and right liver lobe diameter to albumin ratio (RLAR) in the detection of PHG using upper GI endoscopy as a gold standard in patients with liver cirrhosis. Material and Methods: This cross-sectional study was conducted in the Department of Hepatogastroenterology, Sindh Institute of Urology and Transplantation, Karachi. All consecutive patients with ages 18-65 years who were screened using esophagogastroduodenoscopy (EGD) to exclude esophageal varices were enrolled. At the same time, findings related to PHG were noted. After informed consent, all the patients had blood tests including platelet count and albumin and abdominal ultrasound determining spleen diameter and right liver lobe diameter. Results: Out of 111 patients, 59 $(53.15 \%)$ were males with a mean age of $44 \pm 12.61$ years. Rate of PHG was observed in $84.68 \%$ (94/111) cases confirmed by EGD. Sensitivity, specificity, positive predictive value (PPV), negative predictive value (NPV), and accuracy of PSR were $87.23 \%, 5.88 \%, 83.67 \%$, $7.69 \%$, and $74.7 \%$, respectively, and those of RLAR were $28.72 \%, 70.59 \%, 84.38 \%, 15.19 \%$, and $35.14 \%$, respectively. Conclusion: PSR is better predictor of PHG than RLAR but at the expense of relatively lower specificities and NPV likely because of underlying pathophysiology (portal hypertension) which is similar for esophageal varices, PHG, and ascites.

Key words: sensitivity, specificity, gastropathy, endoscopy

\section{INTRODUCTION}

Portal hypertensive gastropathy (PHG) is a complication of liver cirrhosis, characterized by changes in the gastric mucosa of variable severity. It appears endoscopically as "snakeskin-like appearance" of gastric mucosa and mainly involves body and fundus of the stomach. ${ }^{[1]}$ According to Baveno III consensus, it is classified as mild and severe. Mild PHG is defined as mosaic-like appearance without redness of the areola, while severe PHG is manifested by red marks. Furthermore, gastric antral vascular ectasia (GAVE), which is characterized by stripes of red mucosa separated by normal mucosa, is considered a particular pattern of severe
PHG (watermelon stomach), predominantly affects antrum. ${ }^{[2,3]}$

The prevalence of PHG in patients with cirrhosis varies from $11 \%$ to $98 \%$. According to Ahmed et al. and Abbasi et al. from Pakistan, its prevalence was around $80 \%{ }^{[4-7]}$ In patients with cirrhosis, PHG is an important cause of either acute or chronic upper gastrointestinal (GI) blood loss. ${ }^{[8]}$ It accounts for $2-12 \%$ of acute upper GI bleeding, and up to $95 \%$ of cases are related to severe PHG, which can be life threatening, whereas in 3-26\% of cases, it leads to chronic GI bleeding resulting in iron-deficiency anemia. The mortality rate related to PHG bleeding is nearly $12.5 \% .{ }^{[9]}$ According to the study by Kim et al., ${ }^{[10]}$, severe PHG showed 
a significantly high risk of mortality and reduced expected survival time than none or mild PHG.

The gold standard for the diagnosis of PHG is esophagogastroduodenoscopy (EGD). EGD is not only an invasive procedure but it also poses financial and psychological impact on the patient with limited therapeutic options. Thus noninvasive parameters are required to avoid EGD. Ultrasound (US) is widely available imaging modality required in all patients with cirrhosis to identify features of cirrhosis and development of hepatocellular carcinoma. Therefore, the aim of our study was to determine the diagnostic accuracy of platelet count to spleen diameter ratio (PSR) and right liver lobe diameter to albumin ratio (RLAR; noninvasive parameters) in the detection of PHG using upper GI endoscopy as a gold standard in patients with liver cirrhosis.

\section{MATERIAL AND METHODS}

This cross-sectional study was conducted in the Department of Hepatogastroenterology, Sindh Institute of Urology and Transplantation, Karachi. The diagnosis of cirrhosis was established on the basis of any three of the following radiologic features of cirrhosis: altered echo texture of liver, irregular margins, spleen diameter of $>12 \mathrm{~cm}$, portal vein diameter of $>12 \mathrm{~mm}$, and ascites. ${ }^{[11,12]}$ Abdominal ultrasonography was performed to measure the maximum spleen bipolar diameter and right liver lobe diameter in millimeters, using US (TOSHIBA-apleo 50Model MCM17545TS), by single senior radiologist to minimize interobserver variability.

All patients with cirrhosis with ages 18-65 years visiting Outpatient Department of Hepatogastroenterology, SIUT (GI-OPD) were enrolled after informed consent. A blood sample was drawn for complete blood count, creatinine, albumin, bilirubin, alkaline phosphatase, gamma glutamyl transferase, alanine and aspartate aminotransferase, international normalized ratio, and hepatitis viral serology. All enrolled patients were subjected to EGD, and findings related to PHG and varices were recorded. EGD was performed with Olympus GIF-XP180 by single senior endoscopist to curtail interobserver variation.

To avoid bias, patients on treatment with beta blockers, with the history of variceal band ligation, bleeding disorders, renal disease, acute illness, and malignancy and those with portal vein thrombosis were excluded from the study. This study was approved by our institutional ethical committee.

\section{Statistical analysis}

Statistical analysis was performed by IBM-compatible Statistical Package for Social Sciences (SPSS) version 20.0
(SPSS Inc., Chicago, IL, USA). Frequencies and percentages were computed for categorical variables, while quantitative values were presented as mean \pm standard deviation. Sensitivity, specificity, positive predictive value (PPV), negative predictive value (NPV), and diagnostic accuracy of each of the indices PSR and RLAR for the diagnosis of PHG were calculated by taking EGD as gold standard.

\section{RESULTS}

A total of 111 newly diagnosed patients with cirrhosis undergoing screening endoscopy were included in this study. The average age of the patients was $44 \pm 12.61$ years, and other average clinical parameter were also estimated and shown in Table 1. Regarding grade of ascites, 37.84\% were mild, $18.92 \%$ were moderate, and $17.12 \%$ were severe grades of ascites. Rate of PHG was observed in $84.68 \%$ (94/111) of cases confirmed by upper GI endoscopy. Gastric vascular antral ectasia was seen in $17(15.3 \%)$ patients. Hepatitis $\mathrm{C}$ virus was the most common cause of underlying liver disease.

The association of PHG with ascites $(P=0.01)$, esophageal varices $(\mathrm{p}=0.003)$, and Child-Turcotte-Pugh $(\mathrm{CTP})$ class $(P=0.01)$ was statistically significant. However, no statistically significant association of PHG was observed with Model for End-Stage Liver Disease (MELD) score $(P=0.84)$.

Diagnostic accuracy of PSR and RLAR in the detection of PHG in patients with liver cirrhosis is presented in Tables 2 and 3, respectively. Sensitivity, specificity, PPV, NPV, and accuracy of PSR were $87.23 \%, 5.88 \%, 83.67 \%, 7.69 \%$, and $74.7 \%$, and those of RLAR were $28.72 \%, 70.59 \%, 84.38 \%$, $15.19 \%$, and $35.14 \%$, respectively.

\section{DISCUSSION}

About $2-12 \%$ of acute upper GI bleeding cases are related to severe PHG, which can sometimes be life threatening. While up to $26 \%$ patients present with chronic GI blood loss. According to Cremers et al..$^{\left[{ }^{[0]}\right.}$ and Kim et al. ${ }^{[10]}$ severe PHG was significantly associated with higher mortality and reduced expected survival time than none or mild PHG.

This study was aimed to evaluate the diagnostic accuracy of PSR and RLAR to diagnose PHG without endoscopy in patients with liver cirrhosis irrespective of etiology. The frequency of PHG observed in our study was $84.68 \%$ (94/111) with $30.6 \%$ patients having severe PHG. Ahmed $e a l^{[6]}$ and Abbasi et al..$^{[7]}$ from Pakistan found similar prevalence.

According to Hepatitis Antiviral Long-term Treatment against Cirrhosis (HALT-C) trial, albumin and platelets 


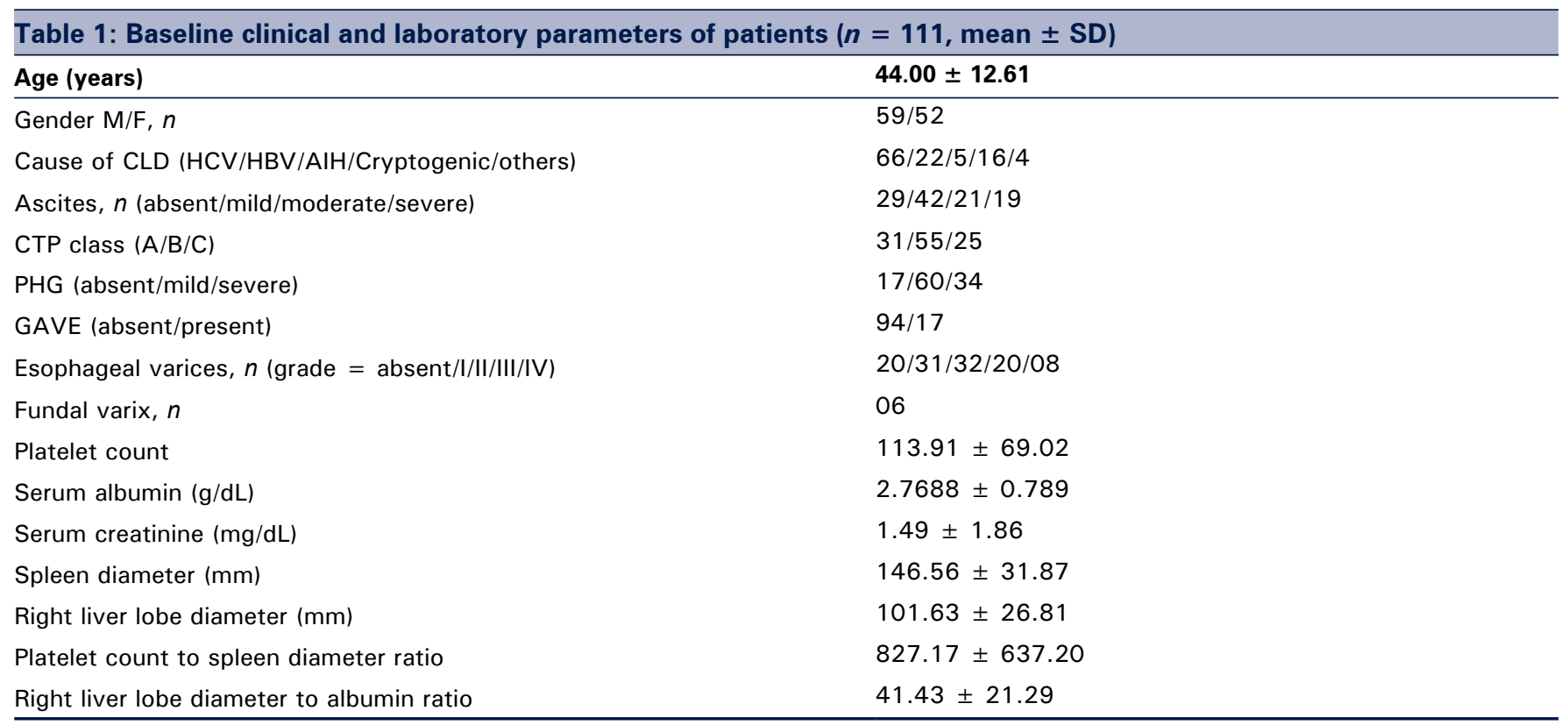

PHG: portal hypertensive gastropathy; AlH: autoimmune hepatitis; CLD: chronic liver disease; CTP: Child-Turcotte-Pugh; F: female; HBV: hepatitis B virus; HCV: hepatitis C virus; M: male; SD: standard deviation.

\begin{tabular}{|c|c|c|c|}
\hline \multirow{2}{*}{$\begin{array}{l}\text { Platelet count to spleen diameter } \\
\text { ratio }\end{array}$} & \multicolumn{2}{|c|}{$\begin{array}{c}\text { Upper GI Endoscopy } \\
\text { (Gold Standard) }\end{array}$} & \multirow[t]{2}{*}{ Total } \\
\hline & Positive & Negative & \\
\hline Positive $\leq 1,326.58$ & 82 (TP) & $16(\mathrm{FP})$ & $98(88.3 \%)$ \\
\hline Negative $>1,326.58$ & $12(\mathrm{FN})$ & $1(\mathrm{TN})$ & $13(11.7 \%)$ \\
\hline \multirow[t]{3}{*}{ Total } & $94(84.7 \%)$ & $17(15.3 \%)$ & 111 \\
\hline & Estimate & \multicolumn{2}{|c|}{ 95\% Confidence Interval } \\
\hline & & Lower & Upper \\
\hline Sensitivity & $87.23 \%$ & $79.0 \%$ & $92.54 \%$ \\
\hline Specificity & $5.88 \%$ & $1.05 \%$ & $26.98 \%$ \\
\hline Positive predictive value & $83.67 \%$ & $75.11 \%$ & $89.69 \%$ \\
\hline Negative predictive value & $7.69 \%$ & $1.37 \%$ & $33.31 \%$ \\
\hline Diagnostic accuracy & $74.77 \%$ & $65.96 \%$ & $81.93 \%$ \\
\hline
\end{tabular}

PHG: portal hypertensive gastropathy; FN: false negative; FP: false positive; TN: true negative; TP: true positive.

\begin{tabular}{|c|c|c|c|}
\hline \multirow[t]{2}{*}{$\begin{array}{l}\text { Right liver lobe diameter to } \\
\text { albumin ratio }\end{array}$} & \multicolumn{2}{|c|}{$\begin{array}{l}\text { Upper GI Endoscopy } \\
\text { (Gold Standard) }\end{array}$} & \multirow[t]{2}{*}{ Total } \\
\hline & Positive & Negative & \\
\hline $\begin{array}{l}\text { Positive } \\
\geq 44.22\end{array}$ & 27 (TP) & $5(\mathrm{FP})$ & $32(28.8 \%)$ \\
\hline $\begin{array}{l}\text { Negative } \\
<44.22\end{array}$ & $67(\mathrm{FN})$ & $12(\mathrm{TN})$ & $79(71.2 \%)$ \\
\hline \multirow[t]{3}{*}{ Total } & $94(84.7 \%)$ & $17(15.3 \%)$ & 111 \\
\hline & Estimate & \multicolumn{2}{|c|}{ 95\% Confidence Interval } \\
\hline & & Lower & Upper \\
\hline Sensitivity & $28.72 \%$ & $20.55 \%$ & $38.56 \%$ \\
\hline Specificity & $70.59 \%$ & $46.87 \%$ & $86.72 \%$ \\
\hline Positive predictive value & $84.38 \%$ & $68.25 \%$ & $93.14 \%$ \\
\hline Negative predictive value & $15.19 \%$ & $8.91 \%$ & $24.7 \%$ \\
\hline Diagnostic accuracy & $35.14 \%$ & $26.89 \%$ & $44.38 \%$ \\
\hline
\end{tabular}

PHG: portal hypertensive gastropathy; FN: false negative; FP: false positive; TN: true negative; TP: true positive. 
count are independently associated with the presence of PHG. HALT-C trial also suggested that PHG is associated with the presence of severe portal hypertension, which is related to high morbidity. ${ }^{[13,14]}$ Similarly, our patients had signs of portal hypertension and liver dysfunction such as enlarged spleen, low platelets, and low serum albumin. We used serum albumin level as a marker of hepatic function in combination with right liver lobe size. The RLAR and PSR are noninvasive parameters that can predict the presence and severity of PHG in patients with liver cirrhosis. ${ }^{[15]}$

Esmat et al. ${ }^{[16]}$ investigated on PSR and RLAR for the detection of varices. None of the study was performed previously to assess noninvasive predictors of PHG. As we did not have any pre-defined cut off values, we used 1,326.58 as cut-off value for the PSR for the detection of PHG, at which sensitivity was $87.23 \%$, specificity $5.88 \%$, PPV $83.67 \%$, NPV $7.69 \%$, and accuracy $74.7 \%$ to determine the PHG. Esmat et al. observed $96.34 \%$ sensitivity, $83.33 \%$ specificity, and 94\% accuracy for detecting the esophageal varices.

Similarly, in this study, cut-off value of 44.25 for RLAR was used to detect PHG. Sensitivity, PPV, NPV, and accuracy of RLAR were $28.72 \%, 70.59 \%, 84.38 \%, 15.19 \%$, and $35.14 \%$, respectively, to detect PHG. Esmat et al. ${ }^{[16]}$ found that sensitivity of RLAR was $91.46 \%$, specificity was $77.78 \%$, and accuracy was $89.00 \%$ to detect the esophageal varices. Our results showed that the PSR is relatively better predictor of PHG than RLAR. However, our study found that these ratios had relatively lower specificities and NPV likely because of underlying pathophysiology (portal hypertension) which is similar for esophageal varices, PHG, and ascites.

According to the research by Kumar et al. . $^{[17]}$ PHG is more often associated with advanced portal hypertension and advanced liver failure. They also found that ascites was significantly associated with PHG. Our study revealed that PSR was found to have diagnostic accuracy of $64.29 \%$ and $77.5 \%$ in relation to mild and moderate/severe ascites, respectively. In accordance to severity of ascites, diagnostic accuracy of RLAR for the detection of PHG was $38.1 \%$ and $42.5 \%$ for mild and moderate/ severe ascites, respectively. When severity of ascites was considered, PSR was found to be a better predictor than RLAR for PHG. Similar to most studies, our study also has few limitations. It is a single center study with small sample size that would limit to extrapolate the present study findings on general population. The identification of these noninvasive parameters is an important clinical goal, thus reducing the need to perform upper GI endoscopy in all patients with liver cirrhosis. To ensure that, patients with PHG are not missed especially with severe PHG, such predictors must have a high sensitivity, even at the expense of a lower specificity. There is need to perform large multicenter center study and to determine cut-off values specific to PHG and our population.

\section{CONCLUSION}

PHG is very common in patients with liver cirrhosis. About one-third of the patients had severe PHG. The presence of ascites, esophageal varices, and CTP class were significantly associated with the presence of PHG. PSR was better predictor of PHG than RLAR but at the expense of relatively lower specificities and NPV likely because of underlying pathophysiology (portal hypertension), which is similar for esophageal varices, PHG, and ascites.

\section{Disclosure}

The authors declare that they have no sources of funding for this study and no conflicts of interest to declare.

\section{REFERENCES}

1. Chung WJ. Management of portal hypertensive gastropathy and other bleeding. Clin Mol Hepatol 2014; 20: 1-5.

2. De Franchis R. Updating consensus in portal hypertension: report of the Baveno III consensus workshop on definitions, methodology and therapeutic strategies in portal hypertension. J Hepatol 2000; 33: 846-52.

3. Elhendawy M, Mosaad S, Alkhalawany W, Abo-Ali L, Enaba M, Elsaka $\mathrm{A}$, et al. Randomized controlled study of endoscopic band ligation and argon plasma coagulation in the treatment of gastric antral and fundal vascular ectasia. United European Gastroenterol J 2016;4: 423-8.

4. Shertsinger AG, Zhigalova SB, Semenova TS, Tsaava DV. [Portal gastropathy: diagnosis, classification]. Eksp Klin Gastroenterol 2012;(6):62-8. [Article in Russian]

5. Gjeorgjievski M, Cappell MS. Portal hypertensive gastropathy: A systematic review of the pathophysiology, clinical presentation, natural history and therapy. World J Hepatol 2016; 8: 231-62.

6. Ahmed S, Mumtaz K, Ahmed US, Shah HA, Abid S, Hamid S, et al. Frequency and characteristics features of portal hypertensive gastropathy with viral cirrhosis. J Coll Physicians Surg Pak 2010; 20: 714-8.

7. Abbasi A, Bhutto AR, Butt N, Munir SM, Dhillo AK. Frequency of Portal Hypertensive Gastropathy and its Relationship with Biochemical, Haematological and Endoscopic Features in Cirrhosis. J Coll Physicians Surg Pak 2011; 21: 723-26.

8. Urrunaga NH, Rockey DC. Portal hypertensive gastropathy and colopathy. Clin Liver Dis 2014; 18: 389-406.

9. Cremers I, Ribeiro S. Management of variceal and nonvariceal upper gastrointestinal bleeding in patients with cirrhosis. Ther Adv Gastroenterol 2014; 7: 206-16.

10. Kim MY, Choi H, Baik SK, Yea CJ, Won CS, Byun JW, et al. Portal Hypertensive Gastropathy: Correlation with Portal Hypertension and Prognosis in Cirrhosis. Dig Dis Sci 2010; 55: 3561-7.

11. Afzal S, Masroor I and Beg M. Evaluation of Chronic Liver Disease: Does Ultrasound Scoring Criteria Help? Int J Chron Dis 2013; ID: 326231. doi:10.1155/2013/326231

12. Shen L, Li JQ, Zeng MD, Lu LG, Fan ST, Bao H. Correlation between ultrasonographic and pathologic diagnosis of liver fibrosis due to chronic virus hepatitis. World J Gastroenterol 2006;12:1292-95.

13. Fontana RJ, Sanyal AJ, Mehta S, Doherty MC, Neuschwander-Tetri BA, 
Everson GT, et al. Portal Hypertensive gastropathy in chronic Hepatitis $\mathrm{C}$ patients with bridging fibrosis and compensated cirrhosis: results from the HALT-C Trial. Am J Gastroenterol 2006; 101: 983-92.

14. Cubillas R, Rockey DC. Portal hypertensive gastropathy: a review. Liver Int 2010; 30: 1094-102.

15. Alempijevic T, Bulat V, Djuranovic S, Kovacevic N, Jesic R, Tomic D, et al. Right liver lobe/albumin ratio: contribution to non- invasive assessment of portal hypertension. World J Gastroenterol 2007; 13: 5331-5.

16. Esmat S, Omarn D, Rashid L. Can we consider the right hepatic lobe size/ albumin ratio, a noninvasive predictor of oesophageal varices in hepatitis C virus-related liver cirrhotic Egyptian patients? Eur J Intern Med 2012; 23: 267-72.

17. Kumar A, Mishra SR, Sharma P, Sharma BC, Sarin SK. Clinical, laboratory, and hemodynamic parameters in portal hypertensive gastropathy: a study of 254 cirrhotics. J Clin Gastroenterol 2010; 44: 294-300.

How to cite this article: Mandhwani R. et al. Noninvasive clinical predictors of portal hypertensive gastropathy in patients with liver cirrhosis. J Transl Intern Med 2017; 5: 169-73. 